\title{
ESTAD O, INTEGRACIÓN Y FUNDAMENTOS TEÓRICOS DE LA IGUALDAD DEMOCRÁTICA
}

\author{
Tomás A. Chuaqui \\ Instituto de Ciencia Politica \\ Pontificia Universidad Católica de Chile
}

\section{CONSIDERACIONES CONCEPTUALES: ¿QUÉ ES "INTEGRAR"?}

La categoría "integración" puede ser usada para referirse a cosas bastante dispares. Existen, en otras palabras, distintos tipos de integración: social, cultural, económica, étnica, racial, sexual, urbana, geográfica-territorial, internacional y política. Interesa, por ende, esclarecer qué tienen en común estos distintos tipos de integración, para aproximar un mayor entendimiento de lo que involucra un esfuerzo integrador por parte del Estado. Es necesario entender lo que significa integrar, para luego determinar por qué la integración es un bien a ser perseguido y, a su vez, para determinar por qué integrar debe ser una función del Estado.

La idea central de esta interpretación del concepto de integración es que está cercanamente ligado a las esperanzas igualitarias de un sistema democrático. En otras palabras, todos, o casi todos, los ámbitos de la integración nacional se remiten a la categoría igualdad. La integración tiene, 0 al menos debería tener, una motivación esencialmente igualitaria, es decir, la intención de establecer y mantener la condición igualitaria de todos los ciudadanos. De esta manera, la integración, en un contexto democrático, está necesariamente ligada a la noción de ciudadanía, y a las esperanzas igualitarias que de ahí se desprenden. Este será el hilo conductor de este trabajo.

Para comenzar, creo importante recordar, que el artículo 1 으 de la Constitución chilena incluye, en su inciso 5ํ, la noción de que es uno de los deberes del Estado "promover la integración armónica de todos los sectores de la Nación". Esta es una innovación constitucional, puesto que este deber del Estado no está contemplado en forma explícita en constituciones chilenas anteriores. Indagando cual era la intención del constituyente se descubre, en varios documentos, que el concepto de "integración" se concibe como coherente con la caracterización del Estado opuesta a la lucha de clases que subyace mucho del proyecto político de la formulación original de la Constitución de 1980; es decir, se utiliza el concepto integración como parte del intento de evitar que el Estado sea concebido como representante necesario de los intereses de una clase en particular. Por supuesto, de esta manera se inscribe en el texto constitucional una teoría social que se aleja abiertamente de toda concepción de la sociedad que la considere caracterizada por lucha de clases, asignándole al Estado una función cuyo objetivo es precisamente reducir o limitar el antagonismo en un sentido muy general. Incluso en el anteproyecto constitucional, el artículo 10 contenía un inciso 60 que decía, en forma explícita: "Se rechaza toda concepción totalitaria de la socie- 
dad o inspirada en el fomento de antagonismos sociales". ${ }^{1}$ Al mismo tiempo, para el constituyente la integración es parte de un proyecto más amplio para alcanzar los (nunca definidos) "grandes y permanentes objetivos de la Nación, resguardar la integridad física y espiritual de la nación, y preservar la identidad histórico-cultural de la Patria". ${ }^{2}$ Esta concepción de la integración superaría la doctrina que considera a la sociedad como el escenario de un enfrentamiento entre clases sociales, y favorecería la consecución del bien común, cuya promoción es también uno de los deberes del Estado contemplado en el artículo 1 oㅡ de la Constitución (Inciso 4 ㅇ). ${ }^{3}$

Obviamente, y especialmente luego de las reformas a la Constitución de 1989 que derogaron el artículo $8 \stackrel{0}{\circ}$, no es indispensable remitirse en forma estricta a las intenciones o motivaciones ideológicas del constituyente en tanto la función integradora del Estado. El inciso 5o de la Constitución es, en las motivaciones del constituyente, parte del intento explícito de la Constitución de 1980 de gestar un sistema político que impida la emergencia de movimientos políticos que pregonen la lucha de clases; es decir, es parte del intento de construir "diques" jurídico-institucionales para proteger al sistema democrático de movimientos que utilizan los mecanismos de la democracia para socavarla. Es una integración a partir de la exclusión. En otras palabras, es uno de los elementos consistentes con el derogado artículo 8 que permanecen en la Constitución. La derogación del artículo 8 a abre la posibilidad, y hace legítima, la interpretación de la función integradora del Estado en forma diferente, o por lo menos ampliando su sentido - cosa que, por lo demás, no es necesariamente inconsistente con las intenciones del constituyente.

Y aquí vale la pena constatar que uno de los peligros permanentes de la noción de integración es, precisamente, y aunque parezca paradójico, la exclusión. Este punto general es independiente de la discusión particular relativa a la posible justificación normativa de la exclusión de movimientos totalitarios, o que se inspiran en el "fomento de antagonismos sociales". Una unidad integrada es casi por definición un objeto que tiene como referente lo que no se integra, lo desunido al objeto. De tal manera que integrar un todo significa siempre, o casi siempre, definir los límites o márgenes, las fronteras, de este todo. El "todo", en este caso, se refiere a la comunidad nacional, a todos los miembros de la comunidad ciudadana chilena.

1 Ver, "Proposiciones e ideas precisas", en Revista chilena de derecho, Vol. 8 № 1-6, enero-diciembre 1981, p. 175, y p. 319. Esta oración desaparece de la versión corregida por el Consejo de Estado del anteproyecto, aunque no se hace referencia a su supresión en el informe enviado al Presidente por este organismo, seguramente por considerársele una enmienda de poca importancia. (Ver, "Informe del Consejo de Estado", en ibid., p. 405.) En todo caso, es razonable suponer que se le consideró redundante dado el contenido del posteriormente derogado artículo 8․ La categoría integración aparece también en el "Preámbulo" a la Constitución que aparecía en el anteproyecto, y que fue suprimido a sugerencia del Consejo de Estado. (Ver, "Texto del Anteproyecto", en ibid., p. 318.) Las razones exactas del Consejo de Estado seguirán siendo un misterio hasta que se hagan públicas las Actas de las reuniones que este organismo sostuvo, aunque se establece que el "Preámbulo" se suprimió "por considerárselo superfluo, toda vez que sus conceptos están incorporados en el capítulo l, sobre bases de la institucionalidad" ("Informe del Consejo de Estado", en ibid., p. 405).

2 Ver, "Proposiciones e ideas precisas", op. cit., p. 168.

3 Ver, "Declaración de principios del gobierno de Chile", en Nuestro Camino, Ediciones Encina, 1976, pp. 296-297; "Objetivo nacional del gobierno de Chile", 1975, pp. 9; 12; 18; 33-34; 41-42; "Discurso de Chacarillas, 1977", en Nueva institucionalidad en Chile: Discursos de S.E. el Presidente de la República General de Ejército D. Augusto Pinochet Ugarte, 1977, p. 13.; "Mensaje Anual 11 Sept. 1977" en ibid., p. 28; "Normas para la nueva Constitución" en Revista chilena de derecho, op. cit., p. 138; "Proposiciones e ideas precisas", op. cit., pp 148; 150; 158; 164; 168; Constitución política de la República de Chile, Art. 1, inciso 5‥ Todos estos documentos evidencian la búsqueda de la configuración de una democracia que sea "autoritaria, protegida, integradora, tecnificada, y de auténtica participación social", en palabras del Presidente Pinochet (énfasis agregado). 
Este es un problema conceptual que tiene consecuencias prácticas. La cuestión recae en donde están, o donde colocar -y a partir de qué criterios- las fronteras de la comunidad ciudadana. Como he dicho, el constituyente de la Constitución de 1980 utilizó un criterio de integración que fue debilitado por las reformas de 1989. Los criterios de integración, por tanto, están desde el punto de vista constitucional, abiertos. Esto además de que es legítimo revisar conceptual y teóricamente qué significa integrar. ¿Qué es lo integrado, y bajo qué principios?

Comenzando negativamente, lo contrario a la integración es la marginalidad, término usualmente asociado a la extrema pobreza; de hecho, el esfuerzo integrador ha sido ligado a la función del Estado en la erradicación de la extrema pobreza. Lo que se persigue es la incorporación a los procesos de desarrollo económico de todos los sectores de la población, un bien difícilmente cuestionable. Más en general, la calidad de miembro pleno en una comunidad nacional es un bien primario a ser distribuido, y es, a la vez, determinante de todo el resto de las decisiones distributivas que tomamos como comunidad política: "determina con quién tomamos estas decisiones, de quién se exige obediencia y se recolectan impuestos, y a quiénes les asignamos bienes y servicios". ${ }^{4}$

En consecuencia, integrar, en un sentido más positivo, significa conformar las partes de un todo. Puesto de esta manera, se presupone que existe un todo -la comunidad nacional- más o menos definible y reconocible al cual deben conformarse las partes. ${ }^{5}$ Al mismo tiempo, parece claro que "integración" contiene una fuerte presuposición igualitaria. Es decir, integrar (particularmente social y políticamente) significa promover igualdad de oportunidades, igualdad de acceso a la participación en los procesos y en las decisiones que afectan a la comunidad nacional. En términos incluso más genéricos, y posiblemente más cercanos a una concepción cultural de la integración, se podría decir que el esfuerzo por gestar una sociedad integrada está ligado al intento de establecer una sociedad en la cual todas sus partes (individuos y asociaciones) se consideran a sí mismas, y son consideradas por las demás, como miembros de una comunidad, y no como extranjeros. ${ }^{6}$

La percepción de sentirse miembro de una comunidad política, el no sentirse como un extranjero en el propio país, sin duda se remite a la categoría igualdad, puesto que se refiere a estar en un pie de igualdad con respecto al Estado, es decir, con respecto al ordenamiento jurídico-institucional y a la ley. ${ }^{7}$ Implica, en otras palabras, la protección de la ley que un Estado le debe, en forma equivalente, a todos los ciudadanos en un régimen democrático de derecho. Un Estado de derecho es aquel en el cual ningún individuo o grupo de individuos está por encima de las leyes y la

$4 \quad$ Walzer, Michael, Spheres of J ustice, Basic Books, 1983, p. 31.

$5 \quad$ No es necesaria, aunque se puede usar, una concepción aristotélica de la comunidad política como un todo anterior a las partes. La concepción aristotélica trae consigo ciertos problemas al ser visto desde la categoría integración, puesto que es precisamente la conformación de este todo, la articulación de las partes de este todo, lo que está en cuestión, y en la práctica el todo se configura en el proceso mismo de integración. Esto tiene particular importancia en las sociedades contemporáneas que se caracterizan por un dinamismo sin precedentes históricos.

6 La definición de quién es extranjero y quién un ciudadano es parte del proceso de integración, y es además particularmente importante dado el proceso de creciente inmigración que se vive actualmente en Chile. Casi está demás decir que la historia socio-política chilena sería incomprensible, y ciertamente incompleta, si no se estudiasen los procesos de integración de las oleadas de inmigrantes cuyos descendientes constituyen parte importante de la población.

7 Esta formulación me fue sugerida por las ideas de Maurizio Viroli, "El significado histórico del patriotismo" y "Nacionalismo y democracia", en Revista de Ciencia Política, Vol. XX, № 1, 1999, pp. 165-194. Ver también del mismo autor, Por amor a la patria, Acento Editorial, 1997. 
constitución. Los reclamos de derecho solo son efectivos si se parte de una igualdad de fuerzas, que es precisamente lo que intenta establecer un régimen democrático de gobierno. ${ }^{8}$ Argumentaré que el concepto de igualdad afín a la integración en sociedades modernas, y necesario para justificar el esfuerzo estatal en esta dirección, es un concepto particular y especificativo.

En relación a la integración, la igualdad puede ser concebida al menos de dos maneras. Se puede entender, en primer lugar, como una exigencia de igualdad, como si fuera un "pago de peaje" antes de ser admitido plenamente a la comunidad nacional. En esta concepción, la comunidad nacional, las partes que la componen, y sus fronteras, estarían predeterminadas por aquellos que ya pertenecen plenamente a ella, y que han recibido adecuada protección de la ley y que, más aún, desde una perspectiva histórica, han sido precisamente aquellos que han excluido (no necesariamente en forma deliberada) a los que se pretende integrar. A esta concepción de igualdad le correspondería, más o menos, la siguiente oferta integradora: "Nos excusamos por haberte excluido en el pasado (suponiendo que se llega a reconocer alguna responsabilidad colectiva de la exclusión); ahora te permitimos (o incluso te ayudamos) a integrarte a nuestra comunidad nacional, es decir, te permitimos (o te ayudamos) a ser como nosotros. En la medida en que lo seas, en que te parezcas a nosotros, puedes ser parte de nuestra comunidad. Ser igual, significa ser como nosotros: de lo que somos culpables es de no haberte permitido en el pasado ser como nosotros, el haber supuesto que eras incapaz o indigno de ser como nosotros. Ahora, que nos hemos iluminado, sabemos que eres capaz y/o digno de ser como nosotros, aunque necesites algo de ayuda para lograrlo, y por eso vamos a hacer un esfuerzo por integrarte, al mismo tiempo que hacemos un esfuerzo por ayudarte a ser similar a nosotros. En la medida en la que seas igual a nosotros, te integramos". ${ }^{9}$

Una concepción alternativa de igualdad más afín a una integración social, cultural y política real requiere la aceptación de la diferencia en relación a este "nosotros" históricamente excluyente. Esta concepción alternativa utiliza una versión más sofisticada de la categoría igualdad, que parte de la premisa de que para que exista una relación igualitaria, no puede ser una de las partes la que defina los términos de la relación integrada, y mucho menos que defina qué es lo que significa ser iguales. Como veremos, esto puede conllevar una reconceptualización de la neutralidad propia de un Estado en un contexto plural. La integración, entonces, no puede justificarse si se la entiende como integración a un objeto preestablecido (siguiendo una lógica de "tómalo o déjalo", "love it or leave it") sino que requiere un reconocimiento de que aquello a lo que se está integrando es como

8 Esto es algo que los ciudadanos de la Atenas democrática sabían muy bien. Nótese lo que dicen, según Tucídides, representantes atenienses a la débil comunidad de Melos: “...vosotros habéis aprendido, igual que lo sabemos nosotros, que en las cuestiones humanas las razones de derecho intervienen cuando se parte de una igualdad de fuerzas, mientras que en caso contrario, los más fuertes determinan lo posible y los débiles lo aceptan". (Tucídides, Historia de la guerra del peloponeso, Libros V-VI, Editorial Gredos, 1992, p. 143.). Este pasaje evidencia la concepción democrática ateniense: el funcionamiento de una democracia pasa por el intento de establecer, legal y jurídicamente, igualdad de fuerzas entre los ciudadanos. Si esta igualdad no existe, sólo la fuerza es fuente de la resolución de disputas, tal como los melios descubren muy a su pesar, quienes son masacrados por el vasto poderío imperial ateniense: "Los atenienses mataron a todos los melios adultos que apresaron y redujeron a la esclavitud a niños y mujeres. Y ellos mismos, con el posterior envío de quinientos colonos, poblaron el lugar". (Ibid., p. 156).

9 Esta concepción de la integración, quizás se caracterizaría mejor bajo la rúbrica "incorporación", o incluso, usando una carga negativa más fuerte, "cooptación". Considero que junto con la exclusión, la cooptación constituye uno de los peligros del esfuerzo integrador. 
es en el presente, al menos en parte, porque se ha excluido ilegítimamente a algunos en el pasado, y, posiblemente, sería notoriamente distinto si hubiera existido integración. Por lo tanto, integración significa transformación, pero no tanto de aquellos que se van integrando, sino del objeto al que se integran (la comunidad nacional), y de los que ya son parte de este todo que se va complementando. Esto no significa que los que se integren deban permanecer intactos, lo cual es un tópico de discusión típico respecto de la integración de culturas indígenas, pues es común que se defienda el mantener en forma intacta a estas culturas. Esto es parte de un argumento un poco distinto: puede ser que sea legítimo proteger y asegurar que se mantenga lo más intacta posible a estas culturas por el valor intrínseco de la diversidad humana, por una "deuda" colectiva incurrida como resultado de ataques injustificados en el pasado, etc. Pero en la medida en que estas culturas intenten integrarse, no se puede exigir transformación unilateral: principalmente los que hayan excluido deben cambiar, pero también aquellos que se integran, aunque sea solo con respecto a la adquisición de capacidades que son indispensables para desempeñarse en la vida nacional. La opción de mantenerse no integrado(s) es, bajo este punto de vista, perfectamente admisible. Pienso que todo esto es consistente con la idea de que el Estado no intente promover una forma específica de bien, de buena vida, en nombre de la cual intente integrar. Es decir, integrar no requiere sacrificar la neutralidad del Estado en tanto la diversidad de concepciones de bien, dado el hecho del pluralismo en las sociedades contemporáneas. Al contrario, integrar significaría que el Estado adopte una posición incluso más neutral, puesto que la exclusión muchas veces se lleva a cabo precisamente en nombre de alguna concepción relativamente específica de una buena vida, favoreciendo en la práctica a aquellos que participan de esta concepción. ${ }^{10}$ En las palabras de Hannah Arendt, algunas de las pocas que profirió aprobatoriamente de la cultura política de Estados Unidos y en las que expresa más bien un deseo que una representación exacta de la realidad norteamericana, "la asimilación no es el precio que hay que pagar por la ciudadanía". ${ }^{11}$

\section{II. ¿POR QUÉ INTEGRAR?}

¿Por qué "integrar"? ¿Qué es lo que se persigue con la integración?. Primariamente, gestar una sociedad en la que todos los ciudadanos la perciban como su hogar; que ningún ciudadano se sienta como un extranjero en su propio país. En forma negativa primero, no es necesario para esto lograr que todos piensen igual, o que todos desarrollen formas de vida similares. Esto significaría darle preferencia a ciertos sectores ciudadanos por sobre los demás, en consonancia con la concepción de igualdad errada que he reseñado más arriba: se le estaría dando preferencia a la forma de vida propia de, o favorecida por, un cierto sector de la población, atribuyéndole mayor veracidad, o superior valor moral. Para evitar esta injerencia social y política ilegítima en la vida de los individuos y de las asociaciones, los derechos individuales y sociales funcionan como guías para delimitar el ámbito adecuado de la intervención estatal y social en la vida de las personas.

10 Para esta concepción de la neutralidad, ver Ronald Dworkin, "Liberalism", en Stuart Hampshire, editor, Public and Private Morality, Cambridge University Press, 1978.

11 Citado en Elisabeth Young-Bruehl, Hannah Arendt, Edicions Alfons el Magnànim-IVEI, 1993 (1982), p. 111. Cabe hacer notar que Arendt expresa estas palabras a modo de una explicación de su decisión de radicarse en Estados Unidos luego de huir de la Alemania nazi. 
Así, el énfasis en derechos individuales no es inmediatamente susceptible a la crítica que los caracteriza como gestores de barreras interpersonales, provocando lo contrario de la integración, es decir, una supuesta separación, o la llamada "atomización" de los individuos, haciendo que la sociedad esté conformada por individuos-islas que persiguen sus propios intereses en forma disgregada. Los derechos individuales sirven, por el contrario, para permitir que se puedan perseguir proyectos de vida disímiles en forma relativamente armónica, dado el hecho del pluralismo. ${ }^{12}$

En primera instancia, creo reconocer dos líneas argumentativas en favor de una mayor integración socio-política, una más bien de principio, siguiendo criterios de justicia social; y una más bien pragmática, que persigue gestar un sistema político más eficiente, eficaz, y estable. Evidentemente existen traslapes entre ambas líneas argumentativas, aunque también es cierto que por momentos pueden estar en una relación de tensión.

Primero, dado el sistema político democrático representativo bajo el cual nos regimos, y el que aspiramos a perfeccionar, las políticas adoptadas e impulsadas por el Estado se legitiman más en la medida en la que son el resultado de amplia participación, y para esto es necesario "integrar" política y socialmente a la población. El sistema político democrático se basa en la noción de que, en principio, todos los ciudadanos son moralmente equivalentes. Es decir, un sistema democrático inclusivo exige un reconocimiento de la capacidad (al menos potencial) de evaluación moral y normativa de todos los ciudadanos. He aquí la singularidad ética de los sistemas democráticos occidentales que emergen del derrumbe histórico e ideológico de proyectos socio-políticos basados en la protección y promoción de privilegios especiales supuestamente debidos a sectores calificados de la población. ${ }^{13}$

Esta idea puede extenderse a la integración ciudadana de trabajadores inmigrantes. Siguiendo un principio de justicia política ofrecido por Michael Walzer, se arguye que "los procesos de autodeterminación a través de los cuales un Estado democrático le da forma a su vida interna, deben estar abiertos, e igualmente abiertos, a todos aquellos hombres y mujeres que viven dentro de su territorio, trabajan en la economía local, y están sujetos a la legislación local". ${ }^{14} \mathrm{Si}$ los ciudadanos de una comunidad democrática están dispuestos a hacer uso del trabajo socialmente útil ofrecido por los inmigrantes, deben estar igualmente dispuestos a extender las fronteras de la ciudadanía para incluir a aquellos que proveen este trabajo socialmente útil. De otra manera, se tiende a gestar una sociedad compuesta por ciudadanos y extranjeros dentro de un mismo territorio, solo los primeros de los cuales gozarían de los beneficios de la ciudadanía los que les permiten definir unilateralmente espacios públicos (jurídicos, institucionales, políticos) de hecho compartidos. Pueden establecerse restricciones a la ciudadanía de inmigrantes, pero éstas no deben ser pétreas ni irrazonablemente estrictas.

Segundo, siguiendo un argumento estilo J ohn Stuart Mill, ${ }^{15}$ más basado ya en el potencial benefi-

12 Este principio está latamente desarrollado en John Rawls, Political Liberalism, Columbia University Press, 1993. Ver también George Kateb, "Individual Rights and Democratic Individuality" en The Inner Ocean: Individualism and Democratic Culture, Cornell University Press, 1992, pp. 1-35.

13 Ver George Kateb, "The Moral Distinctiveness of Representative Democracy", en The Inner Ocean: Individualism and Democratic Culture, Cornell University Press, 1992, pp. 36-56.

14 Walzer, op. cit., p. 60.

15 Ver John Stuart Mill, On Liberty, Penguin Books, 1988. 
cio social de la integración, es imposible predecir de dónde surgirá una buena idea, u opinión. Por lo tanto, es un beneficio para la comunidad en general el que se integre a la participación ciudadana a la mayor parte de la población. Participación e integración no son exactamente lo mismo, pero una mayor integración política y social habilita a la ciudadanía a hacer más efectiva su participación, y además, pragmáticamente hablando, se hace más seguro un sistema político más estable y perdurable en el tiempo si la ciudadanía es partícipe del quehacer nacional.

El problema de las promesas participativas de la democracia se remiten, creo, a la figura clave de Rousseau. La imagen del individuo que se une a todos los demás y, sin embargo, sigue siendo tan libre como antes, persigue las esperanzas no cumplidas de todos los demócratas. ${ }^{16}$ Vivimos bajo sistemas socio-políticos que legitiman la diferenciación social y la desigualdad económica a partir de criterios quizás ingenuamente meritocráticos que buscan premiar el esfuerzo y el talento. Coordinar estas legítimas desigualdades con la igualdad política es, sin duda, uno de los desafíos centrales de toda práctica y teoría democrática, en tanto estas desigualdades tienden a conllevar drásticos diferenciales de poder. Además, a diferencia de lo que se podría argumentar respecto de las democracias antiguas, la evolución de las sociedades modernas ha sido tal que las comunidades ya no son vistas como el foco moral de la vida de los ciudadanos. La individualización, 0 la subjetivización, o la privatización de la vida humana en occidente -cercanamente ligada a la diferenciación social- gesta una fisura moral entre la comunidad y el individuo, colocando en constante sospecha la legitimidad de la autoridad comunitaria sobre los individuos. Al mismo tiempo, las estructuras estatales modernas concentran en sí mismas una autoridad inmensamente superior a lo que era pensado consistente con un régimen democrático en épocas antiguas. Es en estas circunstancias que debe ser pensada la función integradora de un Estado democrático moderno. ${ }^{17}$

\section{III. ¿POR QUÉ DEBE INTEGRAR EL ESTADO?}

La función de integración del Estado es prioritaria con respecto a aquellos sectores de la ciudadanía que han sido injustamente marginados; que no han recibido adecuada protección de la ley; a quienes en la práctica se les ha, limitado su condición ciudadana y tratado como extranjeros en su propio país. Esto es requerido tanto por argumentos de justicia, como por argumentos prácticos. El Estado, en tanto concentración del poder de la comunidad, es el organismo que, aún estando mayormente capacitado para as egurar los iguales derechos ciudadanos de toda la población, ha históricamente fracasado en este deber. Nadie podría negar que ciertos sectores de la ciudadanía, en todas las democracias del mundo, no han recibido igual protección de la ley. Es al Estado, por ende, al que le compete reparar las injusticias del pasado, en nombre de la comunidad toda y, especialmente, en nombre de aquellos que se vieron beneficiados por la marginación de otros. Se invoca un principio de justicia muy básico: el de reciprocidad y el de compensación por beneficios adquiridos ilegítimamente, aunque no necesariamente en forma deliberada. 
El pensar la integración a partir del criterio de compensación no necesariamente se traduce en la adopción de algún sistema de cuotas, o de discriminación positiva en favor de grupos históricamente marginados. La idea de compensación constituye principalmente una lógica para justificar la acción integradora del Estado, acción que, sin duda, exige el uso de recursos que siempre pueden destinarse a objetivos alternativos. Es decir, es un elemento ineludible a considerar en la justificación del esfuerzo colectivo, a través de los mecanismos y recursos estatales, en cuanto a la integración.

A nivel práctico, la integración de sectores tradicionalmente marginados, incorpora a la discusión pública visiones de mundo, opiniones, y necesidades no previsibles, enriqueciendo la discusión, y proporcionando perspectivas diferentes a la resolución de dilemas políticoéticos. Esto es aplicable, por ejemplo, a la incorporación de la juventud y de las mujeres que, ciertamente, poseen una experiencia de lo político parcialmente singular a su grupo, y que no es necesariamente representable por otros sectores. Además, la integración fomenta la participación directa e indirecta de los pares de cada uno de estos sectores tradicionalmente marginados: en la medida en la que aquellos que representan sus opiniones pertenecen al grupo en cuestión, existe una mayor confianza en esta representación, y esto contribuye a la estabilidad del sistema político, a su legitimidad, al afianzamiento de la autoridad pública, etc. No es necesario proveer un argumento estricto en tanto la imposibilidad cuasiepistemológica de conocer y representar las visiones de mundo de grupos a los que uno no pertenezca. Es sin duda posible representar eficazmente a grupos a los que el representante no pertenece, pero solo le es lícito evaluar esta eficacia a los miembros del grupo en cuestión. Aquí se hace referencia, en cierto sentido, a la aspiración democrática de hacer verdaderamente efectiva la condición ciudadana de todos los miembros de la comunidad política.

Bajo este argumento, se justifica el esfuerzo integrador del Estado tanto a grupos injustamente marginados en el pasado, como a grupos que concebiblemente se marginan en forma voluntaria. La integración que se legitima de acuerdo a un principio de beneficio común, en tanto utilidad social a mediano y largo plazo, es aplicable a todos los sectores de la comunidad nacional. Insisto en pensar, eso sí, que el esfuerzo estatal debiera de ser prioritario con respecto a sectores históricamente excluidos en forma ilegítima.

En términos de integración a lo político, las distintas concepciones de la igualdad bosquejadas arriba conllevan consecuencias significativas en relación a las funciones que le competen al Estado. Adoptar la segunda concepción de la igualdad, significa, por ejemplo, que el Estado no puede tomar una posición pasiva al respecto, sino que se ve obligado a tomar una actitud proactiva en búsqueda de la igualdad, que es simultáneamente un prerequisito para, y una expresión de, la integración. De esta manera se le da un sentido concreto, y no excluyente, al requerimiento constitucional en tanto la función integradora del Estado.

En cambio, entender la integración solo como el establecimiento de una vez por todas de una competencia bajo reglas que son verdaderamente neutrales en cuanto a grupos tradicionalmente marginados, ignora el hecho de que las instituciones y los esquemas sociales, fueron, y en gran medida siguen siendo, diseñados por sectores ya integrados, y en respuesta a intereses y motivaciones propias, que no necesariamente corresponden a las motivaciones, intereses y necesidades de aquellos marginados. Por ende, los mismos roles a los que se pretende igualar acceso son definidos por y para los sectores ya integrados. La competencia, entonces, no es igualitaria al 
simplemente aplicar una regla de estricta neutralidad estatal, en lugar de la regla excluyente del pasado. Esta revisión de la neutralidad estatal no la vulnera en tanto distintas concepciones de bien, sino que, al contrario, intenta asegurar que ninguna particular concepción de bien sea esgrimida en forma excluyente por el Estado.

El ejemplo más clásico al respecto, es el hecho que la mayoría de los trabajos no está diseñado para ser ejecutado por alguien que tiene a su cargo el cuidado de un niño. Dado que en las sociedades contemporáneas, en la vasta mayoría de los casos, se espera que sea la mujer la que se dedique al cuidado del bebé, a los hombres les va a ir mejor al competir por empleos que no toman en consideración este aspecto de la vida humana: la reproducción y el cuidado de los niños. ${ }^{18}$ Y esto no es porque exista discriminación abierta. Es simplemente resultado de la forma en la que se establecen los requisitos relevantes para cumplir adecuadamente con los deberes de un empleo: una mujer está menos calificada que un hombre para cumplir sus deberes porque el empleo está diseñado para alguien que está libre de responsabilidades de cuidado infantil. Hay neutralidad sexual (o de género), pero no hay igualdad sexual (o de género) porque el empleo fue diseñado bajo la presuposición de que el empleado tendría a alguien que cuidara de sus hijos, etc. en el hogar. La simple neutralidad no asegura igualdad de oportunidades. De hecho, la mera idea de "neutralidad" requiere ser cuestionada bajo este prisma, puesto que en realidad se está favoreciendo a un sector de la ciudadanía al estar diseñadas las estructuras institucionales con un cierto tipo de persona en mente, que posee características (reales o supuestas) más acordes a estas estructuras institucionales, y a las prácticas sociales que son premiadas económicamente.

En otras palabras, históricamente se ha establecido una incompatibilidad entre el trabajo remunerado y la crianza de niños, provocando desigualdades profundas entre el hombre y la mujer. Como consecuencia, las mujeres tienden a estar en una situación de dependencia económica con respecto al hombre, puesto que por lo general es el hombre el que recibe mayores remuneraciones dentro de una familia o pareja. El enfoque que solamente pretende establecer la neutralidad en cuanto a la evaluación para trabajos es ciego ante estos problemas.

Incluso se podría argumentar que la ausencia de discriminación explícita, es el resultado de que, precisamente, es innecesaria. Las instituciones jurídicas y las prácticas culturalmente establecidas hacen perdurar la situación desmejorada de las minorías étnicas, por ejemplo, sin necesidad de discriminación abierta. A mayor desigualdad social, mayor será el reflejo de intereses de la mayoría étnica en las instituciones sociales, y menor la necesidad de discriminación abierta y arbitraria. ${ }^{19}$ Esto no debe ser interpretado como la búsqueda de una proporción exacta de cada uno de los grupos sociales en instituciones sociales específicas (como las profesiones, o cargos públicos, por ejemplo), sino más bien el intento de impulsar la posición de grupos severamente

18 Esto obviamente también se aplica a las familias en las que se intenta establecer una relación más igualitaria entre los cónyuges en tanto las labores domésticas y la crianza: el hombre que pretenda participar activamente de las labores domésticas y de crianza está en desventaja en relación a otros hombres cuya relación conyugal no es igualitaria. Obviamente, este problema es muy secundario al compararlo con la situación de las mujeres.

19 Para argumentos de este estilo, ver "Difference and Dominance: On Sex Discrimination", capítulo 2 de Catharine MacKinnon, Feminism Unmodified: Discourses on Life and Law, Harvard University Press, 1987, pp. 32-45; Susan Mendus, "La pérdida de la fe: feminismo y democracia" en J ohn Dunn, ed., Democracia: el viaje inacabado (508 a.C-1993), Tusquets, 1995; Carole Pateman, "Críticas feministas a la dicotomía público/ privado", en Carme Castells, ed., Perspectivas feministas en teoría política, Paidós, 1996, pp. 31-52. 
excluidos, con la esperanza de crear un ímpetu lo suficientemente potente como para que la política de integración se haga innecesaria en el futuro.

En este sentido, el problema debe ser redefinido, no ya como un problema de discriminación, sino como un problema de dominación, en el que estructuras jurídico-institucionales que cimientan el poder tienden a favorecer en forma desigual a unos sobre otros. Dado que el problema es de dominación y no de discriminación, la solución tiene que ver con el poder, no con la neutralidad. Lo que se debe perseguir con la integración es una distribución razonablemente equitativa del poder social. La igualdad requiere no solo igualdad de oportunidades con respecto al acceso a ciertos roles definidos por hombres, por ejemplo, sino el poder para definir los roles de acuerdo a estándares femeninos, o de cualquier otro grupo excluido. Baste pensar cómo sería el mundo y la institucionalidad política, por ejemplo, si la predominancia hubiera sido femenina y no masculina, para imaginar las profundas diferencias que existirían respecto del presente en cuanto al tipo de roles valorados públicamente, y premiados en términos de reputación y remuneración económica.

En fin, avanzar en integración pasa por el intento de establecer acceso al poder para alterar los roles mismos. Extendiendo esta idea, la resolución a la problemática excluyente propia de las sociedades crecientemente plurales de la actualidad debe incluir una reconceptualización de la igualdad, siguiendo las líneas bosquejadas anteriormente. La igualdad, según esta reconceptualización, y aunque suene paradójico, no es incompatible con la diferencia. De hecho, el intento de establecer una igualdad sin considerar la diferencia es precisamente lo que ha causado que en las democracias contemporáneas se mantenga la desigualdad y la no integración, aunque se combata en forma muy intensa la discriminación. Para promover igualdad real, en un contexto plural, es necesario entenderla como el reconocimiento de la diferencia, de la legitimidad de la diferencia.

Además, dada la concentración del poder en los aparatos estatales, es solo el agente portador de este poder el que puede promover su dispersión. Integrar, bajo este punto de vista, es consistente con un proceso de descentralización administrativa, y de la autoridad pública. La integración no requiere la concentración del poder; muy por el contrario, la integración de todos los sectores de la nación exige la dispersión del poder. Esto se aplica también a inmigrantes avecindados en el territorio nacional, que en la medida en la que se les impida el acceso a la ciudadanía, son objetos del poder, y marginados de su ejercicio.

Por último, integración cobra una importancia especial durante un período concebido como de "transición", transición de una "nación de enemigos" a una nación, no ya de amigos, pero sí de contrincantes. El quiebre de la institucionalidad democrática chilena tuvo como corolario la disgregación ciudadana, un claro desafiatamiento social en tanto concebir la práctica política como parte de un proyecto común. Le compete al Estado de este período "transitorio" ejercer sus funciones integradoras en forma explícita, no tanto como representante de todos los sectores de la población, pero sí en tanto canalizador y normador de conflictos entre legítimos contendores que dan voz a intereses y concepciones de vida divergentes, que incluyen evaluaciones dispares de la legitimidad histórica del Estado democrático actual. Un ejemplo puede ser, precisamente, la reinterpretación que se ha sugerido anteriormente del sentido mismo del requerimiento constitucional de integración, adecuándolo a un objetivo inclusivo, en lugar de un objetivo excluyente. 


\section{CONCLUSIÓN}

Bajo la perspectiva desarrollada en este trabajo, la función integradora del Estado es fundamental en tanto las aspiraciones de un esquema socio-político democrático, ya que es inseparable de las aspiraciones más tradicionales que sustentan los determinantes igualitarios del concepto de ciudadanía democrático. El modelo de una comunidad legal que se aferra a la autodeterminación a través de la práctica común de ciudadanos asociados tiene como supuesto una cierta concepción de la igualdad que no subsume la diferencia, sino que la asume. Para que la categoría integración adquiera densidad y dirección, es necesario concebirla como una versión de inclusión social, política y cultural, al sistema de reglas jurídico-legales propio de una relación democrática entre el Estado y la sociedad civil. Esta concepción de la integración incluye, por supuesto, la participación en la formulación, perfeccionamiento, y alteración del sistema de reglas jurídico-legales que establecen los términos de la relación estado-sociedad civil, como también los términos de las relaciones públicas entre ciudadanos. Paradójicamente quizás, el pluralismo, y el disenso que surge de éste, pueden, en el contexto de una adecuada concepción de la igualdad democrática, servir como generadores de integración ciudadana: una defensa de la autodeterminación ciudadana de una comunidad de personas libres e iguales contiene necesariamente, a su vez, una defensa implícita (que debería ser siempre explícita) de la exposición a la crítica y a la autocrítica de los mecanismos para establecer, aplicar e implementar reglamentos legales cuyos principios son extensivos a todos. El pluralismo característico de las sociedades modernas, por tanto, puede ser aprovechado como una oportunidad para ampliar el espectro de inclusión, integrando a través de formas de expresión de disenso legalmente institucionalizadas. Como dice Habermas,

La paradójica operación del derecho consiste, por tanto, en que el potencial de conflicto de las libertades subjetivas desencadenadas, el derecho solo lo domestica mediante normas que solamente pueden obligar mientras puedan ser reconocidas como legítimas sobre el vacilante suelo que representan las libertades comunicativas desencadenadas. Un poder que, de otro modo quedaría enfrente de la fuerza sociointegrativa de la comunicación, se convierte así en un medio de integración social al cobrar la forma de acción estatal legítima. Con ello, la integración social cobra una forma particularmente reflexiva: el derecho, al cubrir su necesidad de legitimación con ayuda de la fuerza productiva que representa la comunicación, utiliza como aguijón de discursos públicos jurídicamente institucionalizados, un riesgo de disentimiento convertido ahora en permanente. ${ }^{20}$

Si el Estado ha de integrar, por lo tanto, ha de hacerlo en vistas a una concepción de igualdad democrática que legitima las funciones coercitivas implícitas en un sistema jurídico-legal cuyo resguardo, extensión, e implementación le compete. Integrar, por lo tanto, es democratizar, puesto que es asegurar la condición igualitaria propia de la ciudadanía democrática en un Estado de derecho.

20 Jürgen Habermas, Facticidad y validez: Sobre el derecho y el Estado democrático de derecho en términos de teoría del discurso, Editorial Trotta, 1998 (1992), pp. 661-662. La influencia del pensamiento de Habermas en este trabajo va ciertamente más allá de lo contenido en esta cita, pero sería imposible hacerle justicia en forma plena. 


\section{BIBLIOGRAFÍA}

COMISIÓN DE ESTUDIO DE LA NUEVA CONSTITUCIÓN POLÍTICA DE LA REPÚBLICA, "Proposiciones e ideas precisas", en Revista chilena de derecho, Vol. 8, № 1-6, enero-diciembre 1981, pp. 144-317.

COMISIÓN DE ESTUdIO DE LA NUEVA CONSTITUCIÓN POLÍTICA DE LA REPÚBLICA, "Texto del anteproyecto", en Revista chilena de derecho, Vol. 8, № 1-6, enero-diciembre 1981, pp. 317-375.

CONSEJ O DE ESTADO, "Informe del Consejo de Estado", en Revista chilena de derecho, Vol. 8, № 1-6, enero-diciembre 1981, pp. 376-481.

CONSTITUCIÓN POLÍTICA DE LA REPÚBLICA DE CHILE, Editorial Jurídica, 1998.

DUNN, J OHN, La agonía del pensamiento político occidental, Cambridge University Press, 1996 [1979].

RONALD DWORKIN, "Liberalism", en Stuart Hampshire, editor, Public and Private Morality, Cambridge University Press, 1978, pp. 113-143.

HABERMAS, JÜRGEN, Facticidad y validez: Sobre el derecho y el Estado democrático de derecho en términos de teoría del discurso, Editorial Trotta, 1998 [1992].

KATEB, GEORGE, "Individual Rights and Democratic Individuality", en The Inner Ocean: Individualism and Democratic Culture, Cornell University Press, 1992, pp. 1-35.

KATEB, GEORGE, "The Moral Distinctiveness of Representative Democracy", en The Inner Ocean: Individualism and Democratic Culture, Cornell University Press, 1992, pp. 36-56.

MACKINNON, CATHARINE, Feminism Unmodified: Discourses on Life and Law, Harvard University Press, 1987.

MENDUS, SUSAN, "La pérdida de la fe: feminismo y democracia" en J ohn Dunn, ed., Democracia: el viaje inacabado (508 a.C-1993), Tusquets, 1995

MILL, J OHN STUART, On Liberty, Penguin Books, 1988.

PATEMAN, CAROLE, "Críticas feministas a la dicotomía público/privado", en Carme Castells, ed., Perspectivas feministas en teoría política, Paidós, 1996.

PINOCHET UGARTE, AUGUSTO; MERINO CASTRO, J OSÉ T.; LEIGH GUZMÁN, GUSTAVO; MENDOZA DURÁN, CÉSAR, "Declaración de principios del gobierno de Chile", en Nuestro Camino, Ediciones Encina, 1976, pp. 285-312. PINOCHET UGARTE, AUGUSTO; MERINO CASTRO, J OSÉ T.; LEIGH GUZMÁN, GUSTAVO; MENDOZA DURÁN, CÉSAR, "Objetivo Nacional del gobierno de Chile", 1975.

PINOCHET UGARTE, AUGUSTO, "Discurso en Cerro Chacarillas, con ocasión del Día de la J uventud, el 9 de julio de 1977", en Nueva institucionalidad en Chile: Discursos de S.E. el Presidente de la República General de Ejército D. Augusto Pinochet Ugarte, 1977, pp. 12-15.

PINOCHet UGARTE, AUGUSTO, "Mensaje anual del 11 de septiembre de 1977", en Nueva institucionalidad en Chile: Discursos de S.E. el Presidente de la República General de Ejército D. Augusto Pinochet Ugarte, 1977, pp. 18-29.

PINOCHET UGARTE, AUGUSTO, "Normas para la nueva Constitución”, en Revista chilena de derecho, Vol 8 № 1-6, enerodiciembre 1981, pp. 137-143.

RAWLS, J OHN, Political Liberalism, Columbia University Press, 1993.

ROUSSEAU, J EAN-J ACQUES, El contrato social, Editorial Tecnos, 1995.

TUCíDIDES, Historia de la guerra del peloponeso, Libros V-VI, Editorial Gredos, 1992.

VIROLI, MAURIZIO, "El significado histórico del patriotismo" y "Nacionalismo y democracia”, en Revista de Ciencia Política, Vol. XX, № 1, 1999, pp. 165-194.

VIROLI, MAURIZIO, Por amor a la patria, Acento Editorial, 1997

WALZER, MICHAEL, Spheres of J ustice, Basic Books, 1983.

YOUNG-BRUEHL, ELISABETH, Hannah Arendt, Edicions Alfons el Magnànim-IVEI, 1993 (1982). 


\title{
ESTADO E INTEGRACIÓN: MOVIMIENTOS DE LARGA DURACIÓN, NOCIÓN DE ESTADO Y PREGUNTAS DE INVEST IGACIÓN
}

\author{
Patricio Valdivieso \\ Instituto de Ciencia Politica \\ Pontificia Universidad Católica de Chile
}

Actualmente se reconoce la necesidad de avanzar en la comprensión de las tareas de futuro que debemos emprender en Chile, y ello será posible a partir del entendimiento de las megatendencias que afectan la sociedad chilena y que debieran dar la orientación, a las posibilidades y los límites de la acción colectiva como nación.

En este estudio se identifican y precisan tres de los movimientos de larga duración o megatendencias que están afectando al país, se exponen algunas indagaciones sobre la noción de estado que demanda nuestro tiempo histórico, sobre principios normativos orientadores, y finalmente quedan formuladas algunas preguntas de investigación. En calidad de apéndice, se incluye una selección de trabajos académicos que complementan la perspectiva desarrollada en el estudio.

\section{MOVIMIENTOS DE LARGA DURACIÓN}

El debate nacional en torno a los desafíos del Estado chileno debe considerar las posibilidades y límites de su acción en la etapa de transición hacia el nuevo milenio. De los procesos o tendencias nacionales e internacionales que nos están afectando como sociedad en la actualidad, y que por lo tanto condicionan nuestras acciones, hay por lo menos tres que demandan nuestro reconocimiento: la globalización, el pluralismo, y la democracia. Entonces, cabe partir por precisar el sentido y el alcance de estos conceptos.

\section{Globalización}

La creciente extensión espacial de las relaciones económicas, culturales y políticas, y la progresiva interdependencia de actores y problemas en el mundo, dan lugar a la percepción de un desarrollo global en el planeta. La globalización es resultado de progresos tecnológicos, de los medios de transporte y de las comunicaciones. Pero también van generándose desafíos globales que demandan acciones colectivas favorables a la globalización, tales como los problemas medioambientales y de seguridad internacional, entre otros.

Las fronteras políticas, económicas y culturales de los estados nacionales parecen estar siendo afectadas por el desarrollo global, en mayor medida que en otros tiempos. Diversos actores intergubernamentales y no gubernamentales tienen consecuencias en el sistema internacional. En las relaciones económicas mundiales, se observa una creciente internacionalización de los merca- 